Bull. Soc. math. France

134 (3), 2006, p. 395-415

\title{
SUR LE GROUPE DES CLASSES D'UN SCHÉMA ARITHMÉTIQUE
}

\author{
PAR BRUNO KAHN
}

Avec un appendice de Marc Hindry

\begin{abstract}
RÉSUmÉ. - Nous donnons une démonstration du fait que le groupe des classes d'un schéma irréductible de type fini sur $\operatorname{Spec} \mathbf{Z}$ est de type fini. Cette preuve ne repose pas sur le théorème de Mordell-Weil-Néron, mais plutôt sur le théorème de Mordell-Weil classique, le théorème de Néron-Severi et les théorèmes de Hironaka et de Jong sur la résolution des singularités. Nous en déduisons quelques corollaires, parmi lesquels le théorème de Mordell-Weil-Néron lui-même.

AbSTRACT (On the class group of an arithmetic scheme). - We present a proof that the class group of an irreducible scheme of finite type over $\operatorname{Spec} \mathbf{Z}$ is finitely generated. This proof does not rely on the Mordell-Weil-Néron theorem but rather on the classical Mordell-Weil theorem, the Néron-Severi theorem and Hironaka and de Jong's theorems on resolution of singularities. We derive some corollaries, including the Mordell-Weil-Néron theorem itself.
\end{abstract}

Texte reçu le 15 février 2005, révisé le 20 juillet 2005, accepté le 4 octobre 2005.

Bruno Kahn, Institut de Mathématiques de Jussieu, 175-179 rue du Chevaleret, 75013 Paris (France). - E-mail : kahn@math.jussieu.fr

MARC Hindry, Institut de Mathématiques de Jussieu, 175-179 rue du Chevaleret, 75013

Paris (France). - E-mail : hindry@math.jussieu.fr

Classification mathématique par sujets (2000). — 11G99, 14F22, 14E15, 14G99, 14K99.

Mots clefs. - Géométrie arithmétique, résolution des singularités, variétés abéliennes, groupe des classes, groupe de Brauer. 


\section{Introduction}

Soit $X$ un schéma noethérien. Rappelons (voir [13, §21], [15, ch. II, §6, p. 131 et 143]) la définition du groupe des classes $\mathrm{Cl}(X)$ et du groupe de $\mathrm{P} i$ card $\operatorname{Pic}(X)$ :

- $\mathrm{Cl}(X)$ est le quotient du groupe des cycles de codimension 1 de $X$ par le sous-groupe des cycles principaux.

- Pic $(X)$ est le groupe des classes d'isomorphisme de fibrés en droite (ou de $\mathcal{O}_{X}$-modules inversibles) sur $X$.

On a aussi le groupe quotient $\operatorname{Div}(X) / \operatorname{Div}^{p}(X)$ des diviseurs de Cartier par les diviseurs de Cartier principaux. La relation entre ces trois groupes est donnée par le diagramme suivant

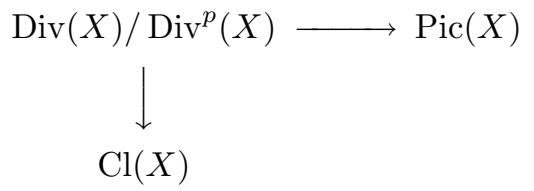

où :

- l'homomorphisme horizontal est injectif, et bijectif si par exemple $X$ est réduit $[13,21.3 .4]$ ou quasi-projectif sur une base affine (ibid., 21.3.5);

- l'homomorphisme vertical est injectif si $X$ est normal et bijectif si et seulement si $X$ est localement factoriel, par exemple régulier ( $c f .[13,21.6 .10]$, $[15$, p. 142 , rem. 6.11 .2 et cor. 6.16$])$.

Si $U$ est un ouvert schématiquement dense de $X$, on a la suite exacte bien connue :

$$
0 \rightarrow \Gamma\left(X, \mathbb{G}_{m}\right) \longrightarrow \Gamma\left(U, \mathbb{G}_{m}\right) \longrightarrow \bigoplus \mathbf{Z} \rightarrow \mathrm{Cl}(X) \longrightarrow \mathrm{Cl}(U) \rightarrow 0
$$

où la somme (finie) porte sur les points de codimension 1 de $X$ qui ne sont pas dans $U$.

Finalement, si $X$ est de dimension $d$ on a aussi le groupe $\mathrm{CH}_{d-1}(X)$ des cycles de dimension $d-1$ sur $X$ modulo l'équivalence rationnelle [9, ch. 2], qui coïncide avec $\mathrm{Cl}(X)$ si $X$ est irréductible.

Le but de ce travail est de présenter une démonstration des énoncés suivants :

THÉORÈme 1. - Supposons $X$ de type fini sur $\operatorname{Spec} \mathbf{Z}$.

a) Si $X$ est réduit, le groupe $\Gamma\left(X, \mathbb{G}_{m}\right)$ est de type fini.

b) Si $X$ est irréductible, le groupe $\mathrm{Cl}(X)$ est de type fini.

Corollaire 1. - Si X est normal et de type fini sur $\operatorname{Spec} \mathbf{Z}, \operatorname{Pic}(X)$ est de type fini.

Corollaire 2 (cf. Guralnick et al. [14, prop.6.1]). - Le groupe $\operatorname{Cl}(X)$ (resp. Pic $(X))$ est de type fini si $X$ est irréductible (resp. normal) et de type fini sur $\operatorname{Spec} k$, où $k$ est un corps de type fini sur son sous-corps premier.

TOME $134-2006-\mathrm{N}^{\mathrm{O}} 3$ 
En effet, on peut choisir un modèle $U$ de $k$, irréductible et de type fini sur Spec $\mathbf{Z}$, et (quitte à rétrécir $U$ ) épaissir $X$ en $\mathcal{X}$ irréductible (resp. normal) et de type fini sur $U$. Alors $\mathcal{X}$ est de type fini sur $\operatorname{Spec} \mathbf{Z}$ et $\mathrm{Cl}(\mathcal{X}) \rightarrow \operatorname{Cl}(X)$ est surjectif.

COROllaire 3 (Néron [29]). - Soit $k$ un corps de type fini sur son sous-corps premier, et soit $A$ une variété abélienne sur $k$. Alors le groupe $A(k)$ est de type fini.

Démonstration. — On applique le corollaire 2 à la variété abélienne duale $A^{\vee}$ de $A$, en notant que $\operatorname{Pic}^{0}\left(A^{\vee}\right)=A(k)$.

Ainsi, pour obtenir le corollaire 3, il n'est pas nécessaire d'utiliser la théorie de la $K / k$-trace, ni de faire une deuxième fois (géométrique) la démonstration (arithmétique) du théorème de Mordell-Weil. Malheureusement je ne sais pas déduire du théorème 1 le théorème général de Lang-Néron [20] :

ThÉORÈme 2. - Si K/k est une extension régulière de type fini et si $A$ est une $K$-variété abélienne, alors le groupe $A(K) / A_{0}(k)$ est de type fini, où $A_{0}$ est la $K / k$-trace de $A$.

On trouvera dans l'appendice A des rappels sur la $K / k$-trace et dans l'appendice $B$ une démonstration de ce théorème (et même d'un énoncé un peu plus général), rédigée par Marc Hindry dans le langage des schémas.

Voici également deux énoncés liés aux précédents : le premier étend le théorème de Néron-Severi rappelé ci-dessous (théorème 5).

ThÉORÈme 3. - Soient $k$ un corps algébriquement clos et $X$ une $k$-variété de dimension $\leq d$. Alors le groupe $A_{d-1}(X)$ des cycles de dimension $d-1$ sur $X$ modulo l'équivalence algébrique est de type fini.

THÉORÈme 4 . - Soient $k$ un corps, $K$ un corps de type fini sur $k$ et $L / K$ une extension de type fini, avec $K$ algébriquement fermé dans L. Soit p l'exposant caractéristique de $k$. Faisons l'une des hypothèses suivantes :

(i) $k$ est un corps de nombres.

(ii) $H^{1}(k, A)$ est fini pour tout module galoisien A fini d'ordre premier à $p$. Exemples : $k$ algébriquement clos, fini, local, corps de séries formelles itérées sur un tel corps,...

Alors la partie première à $p$ de $\operatorname{Ker}(\operatorname{Br}(K) \rightarrow \operatorname{Br}(L))$ est finie, où $\mathrm{Br}$ désigne le groupe de Brauer.

\section{REMARQUES 1}

1) Dans le cas affine, le théorème 1,a) est dû à Samuel [32, th. 1] (et la démonstration que nous donnons est essentiellement la sienne).

2) Lorsque $X$ est normal, le théorème 1, b) est en principe dû à Roquette [30] (voir la note en bas de page). Roquette procède par récurrence sur la dimension 
de $X$ et utilise la thèse de Néron généralisant le théorème de Mordell-Weil (corollaire 3 ci-dessus), ainsi que la génération finie du groupe de Néron-Severi (théorème 5 ci-dessous) $^{(1)}$.

3) Le théorème 1, a) est faux si on ne suppose pas $X$ réduit. Exemple bien connu : $X=\operatorname{Spec} A$, avec $A=A_{0}[\epsilon] / \epsilon^{2}$ où $A_{0}=\mathbf{Z}[t]$ ou $\mathbf{F}_{p}[t]$.

4) Le théorème $1, b)$ est faux si on ne suppose pas $X$ irréductible. En effet, si $X=Z_{1} \cup Z_{2}$, où $Z_{1}$ et $Z_{2}$ sont deux sous-schémas fermés dont les supports sont différents du support de $X$, on a une suite exacte "de Mayer-Vietoris"(2)

$$
\begin{aligned}
0 \rightarrow \Gamma\left(X, \mathbb{G}_{m}\right) \longrightarrow \Gamma\left(Z_{1}, \mathbb{G}_{m}\right) & \oplus \Gamma\left(Z_{2}, \mathbb{G}_{m}\right) \\
& \longrightarrow \Gamma\left(Z_{1} \cap Z_{2}, \mathbb{G}_{m}\right) \stackrel{\partial}{\longrightarrow} \mathrm{Cl}(X)
\end{aligned}
$$

Choisissons $X=\operatorname{Spec} B$, avec $B=A_{0}[x, y] / x\left(y^{2}-x\right)$ où $A_{0}$ est comme en a), et $Z_{1}, Z_{2}$ donnés respectivement par les équations $x=0$ et $y^{2}=x$, de sorte que $Z_{1} \cap Z_{2}=\operatorname{Spec} A$ où $A$ est comme dans la remarque précédente. Alors $\Gamma\left(Z_{1}, \mathbb{G}_{m}\right)$ et $\Gamma\left(Z_{2}, \mathbb{G}_{m}\right)$ sont finis. D'autre part, $\Gamma\left(Z_{1} \cap Z_{2}, \mathbb{G}_{m}\right)$ n'est pas de type fini comme vu dans la remarque précédente, et donc $\mathrm{Cl}(X)$ non plus.

Par contre, on déduit facilement du théorème $1, \mathrm{~b})$ que $\mathrm{CH}_{d-1}(X)$ est de type fini pour $X$ quelconque, grâce aux suites exactes du type

$$
\mathrm{CH}_{d-1}\left(Z_{1}\right) \oplus \mathrm{CH}_{d-1}\left(Z_{2}\right) \longrightarrow \mathrm{CH}_{d-1}(X) \rightarrow 0
$$

si $X$ est réunion de deux fermés $Z_{1}$ et $Z_{2}$.

5) Le rapporteur m'a fait remarquer que le théorème 1 se généralise au cas d'un $X$-tore $T$ en se ramenant à ce théorème : pour la génération finie de $H^{1}(X, T)$ il faut supposer $X$ normal. Dans ce cas, on utilise le fait que $T$ est déployé par un morphisme fini étale (surjectif) d'après [7, ch. X, cor. 5.14]. Le rapporteur observe par contre que la version torique du corollaire 2 est fausse en général, déjà avec $X=\operatorname{Spec} \mathbf{Q}$ et $T=R_{\mathbf{Q}(\sqrt{-1}) / \mathbf{Q}}^{1} \mathbb{G}_{m}$. (Elle est vraie pour les tores flasques au moins sur les corps de type fini d'après ColliotThélène \& Sansuc, [5, p. 192, th. 1] ; leur démonstration repose d'ailleurs sur le théorème 1 !)

\footnotetext{
${ }^{(1)}$ La démonstration de Roquette offre toutefois une difficulté en caractéristique $p:$ si $L / K$ est une extension de type fini de degré de transcendance 1 , où $K$ et $L$ sont de type fini sur $\mathbf{F}_{p}$ et $K$ est algébriquement fermé dans $L$, il n'est pas vrai en général que la $K$-courbe projective régulière de corps des fonctions $L$ soit lisse sur $K$. Sa variété de Picard n'est donc pas en général une variété abélienne et le théorème de Néron ne peut pas être invoqué tel quel, cf. exemple 1. L'approche du présent article contourne cet écueil. Il serait intéressant de trouver une démonstration du théorème de Roquette qui en caractéristique $p$ évite le recours au théorème de de Jong.

${ }^{(2)}$ Sans établir cette suite exacte, décrivons l'homomorphisme $\partial$. Soit $u$ une unité sur $Z_{1} \cap Z_{2}$. Relevons $u$ en deux "fonctions méromorphes" $f_{1}$ et $f_{2}$ sur $Z_{1}$ et $Z_{2}$. Les cycles associés à $f_{1}$ et $f_{2}$ se recollent en un cycle sur $X$, d'où $\partial(u) \in \mathrm{Cl}(X)$.
}

TOME $134-2006-\mathrm{N}^{\mathrm{O}} 3$ 
6) Le corollaire 1 est faux si on retire l'hypothèse "normal". Par exemple, si $X$ est la courbe projective intègre donnée par l'équation $y^{2} z=x^{3}$ sur un anneau de base $A_{0}$ comme en a), alors $\operatorname{Pic}(X) \rightarrow \mathrm{Cl}(X)=\mathbf{Z}$ est surjectif de noyau $A_{0}, c f$. [9, ex. 2.1.2].

7) Dans le corollaire $2, \Gamma\left(X, \mathbb{G}_{m}\right)$ n'est bien sûr pas de type fini (à moins que $k$ ne soit fini), mais le groupe $\Gamma\left(X, \mathbb{G}_{m}\right) / k^{*}$ l'est si $X$ est géométriquement intègre, par la méthode du $§ 2.1$ ci-dessous.

8) Dans le théorème 4, l'hypothèse "algébriquement fermé" est nécessaire : l'énoncé est faux en général pour une extension finie, par exemple pour $\mathbf{Q}(\sqrt{-1}) / \mathbf{Q} \ldots$ En fait, pour une extension finie non triviale $L / K$ de corps globaux, $\operatorname{Ker}(\operatorname{Br}(K) \rightarrow \operatorname{Br}(L))$ est toujours infini par un théorème de Fein, Kantor et Schacher [8]. Il y a de nombreux autres exemples.

9) Le théorème 4, (ii) est nettement plus élémentaire que le théorème 1 : il ne fait intervenir que le théorème de Mordell-Weil faible.

10) Dans le théorème 4, (ii) j'ignore ce qu'il advient de la $p$-torsion quand (par exemple) $k$ est algébriquement clos de caractéristique $p$. D'autre part, supprimant toute hypothèse sur $k$, je ne suis pas parvenu à trouver d'exemple d'extension de type fini $L / K$ (disons, en caractéristique zéro) avec $K$ algébriquement fermé dans $L$ et telle que $\operatorname{Ker}(\operatorname{Br}(K) \rightarrow \operatorname{Br}(L))$ soit infini.

ExEMPLE 1. - Sur $k=\mathbf{F}_{p}(t)$, soit $X$ la courbe projective d'équation $x^{2} z^{p-2}=y^{p}-t z^{p}$. Alors $X$ est régulière sans être lisse sur $k$. D'après le corollaire $2, \operatorname{Pic}(X)$ est de type fini bien que la variété de Picard de $X$ contienne un sous-groupe unipotent non trivial.

Les ingrédients de la démonstration du théorème 1 sont les suivants :

1) Le fait, rappelé ci-dessous, que le groupe de Néron-Severi d'une variété projective lisse sur un corps algébriquement clos est de type fini.

2) L'existence de la variété de Picard d'une variété projective lisse $X$ sur un corps $k$, définie sur $k$, "représentant" le groupe $\mathrm{Cl}^{0}(X)=\operatorname{Pic}^{0}(X)$ des classes de diviseurs algébriquement équivalents à 0 .

3) Le théorème de Mordell-Weil : le groupe des points rationnels d'une variété abélienne sur un corps de nombres est de type fini.

4) Les théorèmes classiques de finitude en théorie algébrique des nombres : finitude du groupe des classes et théorème des unités de Dirichlet.

5) Des résultats de désingularisation : théorème de de Jong [17, th. 4.1] en caractéristique $p$ et résolution des singularités de Hironaka [16] en caractéristique 0 . (En caractéristique 0 on pourrait se contenter du théorème de de Jong, plus élémentaire.)

Le résultat 2) est dû à de nombreux auteurs : Matsusaka [22], Chow [4], Weil (reproduit dans Lang [18, ch. VI]) et a bien sûr été complètement réécrit par Grothendieck et ses successeurs [11] (voir aussi [2, ch. 8 et 9]) : nous n'aurons 
besoin que d'une version naïve $[18$, p. 148, th. 1]. Notons seulement que, la variété d'Albanese $A$ de $X$ étant construite, la variété de Picard de $X$ n'est autre que celle de $A$, c'est-à-dire la variété abélienne duale de $A$. (Grothendieck, quant à lui, procède dans l'ordre inverse.)

Je remercie Luca Barbieri-Viale, Marc Hindry, le rapporteur et tout particulièrement Jean-Louis Colliot-Thélène pour leurs commentaires sur les premières versions de ce texte. C'est Marc Hindry qui m'a convaincu d'énoncer le théorème 1 dans la plus grande généralité.

\section{Le groupe de Néron-Severi}

Soient $k$ un corps algébriquement clos et $X$ une $k$-variété projective lisse. Le groupe de Néron-Severi de $X$ est le quotient

$$
\operatorname{NS}(X)=\operatorname{Pic}(X) / \operatorname{Pic}^{0}(X)
$$

où $\operatorname{Pic}^{0}(X)$ est le sous-groupe $\operatorname{de} \operatorname{Pic}(X)=\mathrm{Cl}(X)$ formé des classes de diviseurs algébriquement équivalents à 0 .

ThÉorème 5 (Néron-Severi [29], [36], [35]). — NS(X) est un Z-module de type fini.

La démonstration de ce théorème est facile en caractéristique 0 grâce à la théorie de Hodge : en utilisant l'invariance de $\mathrm{NS}(X)$ par changement de base algébriquement close, on peut d'abord se ramener au cas où $k$ est la clôture algébrique d'un corps de type fini sur $\mathbf{Q}$, puis plonger $k$ dans $\mathbf{C}$; le résultat suit alors de l'injection

$$
\mathrm{NS}(X) \hookrightarrow H_{\mathrm{an}}^{2}(X, \mathbf{Z})
$$

qui résulte de la suite exacte de l'exponentielle $0 \rightarrow \mathbf{Z} \rightarrow \mathcal{O}_{X}^{\text {an }} \rightarrow\left(\mathcal{O}_{X}^{\text {an }}\right)^{*} \rightarrow 0$ (cf. [15, p. 447]).

Si $X$ est une variété abélienne en caractéristique quelconque, le résultat suit facilement de l'injection

$$
\mathrm{NS}(X) \hookrightarrow \operatorname{Hom}_{k}\left(X, X^{\vee}\right)
$$

où $X^{\vee}$ est la variété abélienne duale de $X$, la génération finie du groupe de droite étant obtenue par un argument $\ell$-adique et un argument de hauteur [27, p. 177].

En caractéristique quelconque et pour $X$ général, il y a plusieurs démonstrations d'esprits très différents :

1) Dans celle de Lang et Néron [20], le résultat est déduit du théorème 2 ci-dessus par un joli argument géométrique : elle est reproduite dans Lang [19].

2) Pour une surface, on trouve une démonstration dans Milne [24, ch. V, th. 3.25]. Elle se découpe en deux morceaux :

TOME $134-2006-\mathrm{N}^{\mathrm{O}} 3$ 
a) Le groupe $\mathrm{N}(X):=\mathrm{NS}(X) /$ tors est libre de rang fini (ibid., lemme 3.26), ce qui est obtenu par un argument $\ell$-adique; on pourrait remplacer cette démonstration par une qui utiliserait le théorème de l'indice de Hodge (Segre [34], Grothendieck [12], voir [15, ch. V, th. 1.9]).

b) La torsion de $\mathrm{NS}(X)$ est finie [24, ch. V, lemme 3.27], ce qui est obtenu grâce au théorème de Riemann-Roch et à la théorie des variétés de Chow.

3) Pour $X$ de dimension quelconque, Milne démontre que $\mathrm{N}(X)$ est de type fini dans [24, ch. VI, th. 11.7], toujours par un argument $\ell$-adique (on notera que sa démonstration est incomplète), mais ne traite pas la torsion de $\operatorname{NS}(X)$.

4) Toujours dans le cas général, on pourrait se ramener au cas d'une surface grâce à des théorèmes de Lefschetz faibles, mais il est plus simple de raisonner comme dans [1, Exp. XIII, th. 5.1], qui donne à mon avis la raison la plus claire pour laquelle le théorème est vrai :

a) $\mathrm{N}(X)$ est libre de type fini (ibid., prop. 5.2, argument $\ell$-adique, tout à fait complet, $c f$. aussi [9, ex. 19.1.4]).

b) $\operatorname{Pic}^{\tau}(X):=\left\{x \in \operatorname{Pic}(X) \mid \exists n>0: n x \in \operatorname{Pic}^{0}(X)\right\}$ est représentable par un schéma en groupes de type fini, donc n'a qu'un nombre fini de composantes connexes.

\section{Démonstration du théorème 1}

\subsection{Démonstration de a). - On a d'abord :}

Lemme 1. - Soit $f: X \rightarrow S$ un morphisme compactifiable : $X$ se plonge au-dessus de $S$ comme ouvert dense dans un $S$-schéma propre $\pi: \bar{X} \rightarrow S$. Supposons de plus $X$ réduit. Soient $Z$ l'image de $\pi$ et $\widetilde{Z}$ le normalisé de $Z$ dans $\pi$. Alors le groupe $\Gamma\left(X, \mathbb{G}_{m}\right) / \Gamma\left(\widetilde{Z}, \mathbb{G}_{m}\right)$ est de type fini.

D'après Nagata [28], l'hypothèse est vérifiée au moins si $S$ est le spectre d'un corps ou de $\mathbf{Z}$.

Démonstration. - La suite exacte (2) nous ramène à $X$ irréductible; l'hypothèse reste vérifiée. La suite exacte $(1)$, appliquée à $(X, U) \equiv(\bar{X}, X)$, nous ramène à démontrer l'assertion pour $\bar{X}$; mais on a $\Gamma\left(\widetilde{Z}, \mathbb{G}_{m}\right) \stackrel{\sim}{\longrightarrow} \Gamma\left(\bar{X}, \mathbb{G}_{m}\right)$.

Pour démontrer le théorème 1, a), revenons au cas où $S=\operatorname{Spec} \mathbf{Z}$. Si $\pi$ n'est pas surjectif, $Z$ et $\widetilde{Z}$ sont des corps finis et la conclusion est claire. Si $\pi$ est surjectif, $\widetilde{Z}$ est le spectre d'un ordre dans un anneau d'entiers de corps de nombres et la conclusion résulte du théorème des unités de Dirichlet. 
2.2. Démonstration de b). — Comme évidemment $\mathrm{Cl}(X)=\mathrm{Cl}\left(X_{\text {red }}\right)$, on peut supposer $X$ réduit, c'est-à-dire intègre.

LEMme 2. - Soit $X$ comme dans le théorème $1, \mathrm{~b})$, et soit $U$ un ouvert non vide de $X$. Alors le théorème est vrai pour $X$ si et seulement s'il est vrai pour $U$.

Démonstration. - Cela résulte de la suite exacte (1).

Lemme 3. - Pour démontrer le théorème 1, b), on peut supposer $X$ régulier.

En effet, $X$ contient un ouvert régulier non vide d'après [13, cor. 6.12.6]; l'assertion résulte donc du lemme 2.

À partir de maintenant, on suppose $X$ régulier.

Proposition 1. - Soit $f: Y \rightarrow X$ un revêtement étale. Si $\operatorname{Pic}(Y)$ est de type fini, alors $\operatorname{Pic}(X)$ est de type fini.

Démonstration. — Comme $X$ est régulier, on a

$$
\mathrm{Cl}(X)=\operatorname{Pic}(X)=H_{\text {ét }}^{1}\left(X, \mathbb{G}_{m}\right)
$$

et de même pour $Y$. Un calcul élémentaire montre que $\operatorname{Ker}(\operatorname{Pic}(X) \rightarrow \operatorname{Pic}(Y))$ s'injecte dans l'homologie du complexe

$$
\Gamma\left(Y, \mathbb{G}_{m}\right) \longrightarrow \Gamma\left(Y \times_{X} Y, \mathbb{G}_{m}\right) \longrightarrow \Gamma\left(Y \times_{X} Y \times_{X} Y, \mathbb{G}_{m}\right)
$$

les différentielles étant données par la somme alternée des projections partielles. (En fait on a même égalité.) D'après $2.1, \Gamma\left(Y \times_{X} Y, \mathbb{G}_{m}\right)$ est de type fini et on conclut.

Remarque 2. - Dans le cas où $f$ est galoisien de groupe $G$, ce raisonnement revient à utiliser la suite exacte résultant de la suite spectrale de HoschschildSerre

$$
0 \rightarrow H^{1}\left(G, \Gamma\left(Y, \mathbb{G}_{m}\right)\right) \longrightarrow \operatorname{Pic}(X) \longrightarrow \operatorname{Pic}(Y)^{G} \text {. }
$$

LEMME 4. - Soient $k$ un corps fini ou un corps de nombres et $Y$ une $k$-variété projective lisse géométriquement intègre. Notons $\bar{k}$ une clôture séparable de $k$, $G=\operatorname{Gal}(\bar{k} / k)$ et $\bar{Y}=Y \otimes_{k} \bar{k}$. Alors les groupes $\operatorname{Pic}(\bar{Y})^{G}$ et $\operatorname{Pic}(Y)$ sont de type fini.

Démonstration. — On déduit de la suite exacte

$$
0 \rightarrow \operatorname{Pic}^{0}(\bar{Y}) \longrightarrow \operatorname{Pic}(\bar{Y}) \longrightarrow \mathrm{NS}(\bar{Y}) \rightarrow 0
$$

une suite exacte

$$
0 \rightarrow \operatorname{Pic}^{0}(\bar{Y})^{G} \longrightarrow \operatorname{Pic}(\bar{Y})^{G} \longrightarrow \mathrm{NS}(\bar{Y})
$$

Or $\operatorname{Pic}^{0}(\bar{Y})=P(\bar{k})$, où $P$ est la variété de Picard de $X$, définie sur $k$, donc $\operatorname{Pic}^{0}(\bar{Y})^{G}=P(k)$ est fini si $k$ est fini et de type fini si $k$ est un corps de nombres d'après le théorème de Mordell-Weil [21], et celui de droite est de type

TOME $134-2006-\mathrm{N}^{\mathrm{O}} 3$ 
fini par le théorème de Néron-Severi (théorème 5). Comme $\operatorname{Pic}(Y) \hookrightarrow \operatorname{Pic}(\bar{Y})^{G}$ (Hilbert 90), $\operatorname{Pic}(Y)$ est aussi de type fini.

On distingue maintenant deux cas :

1) Le morphisme $g: X \rightarrow \operatorname{Spec} \mathbf{Z}$ n'est pas dominant.

Alors $X$ est régulier sur $\operatorname{Spec} \mathbf{F}_{p}$ pour un nombre premier $p$ convenable, donc lisse puisque $\mathbf{F}_{p}$ est parfait. D'après de Jong [17, th. 4.1], un ouvert non vide convenable de $X$ est revêtu (via un morphisme fini étale) par un ouvert d'une $\mathbf{F}_{p}$-variété projective lisse intègre $Y$. D'après 2.1 , le lemme 2 et la proposition 1 , il suffit de démontrer que $\operatorname{Pic}(Y)$ est de type fini, ce qui résulte du lemme 4.

2) Le morphisme $g: X \rightarrow \operatorname{Spec} \mathbf{Z}$ est dominant.

Soit $X_{\mathbf{Q}}$ la fibre générique de $g$ : par la résolution des singularités de Hironaka [16], un ouvert affine de $X_{\mathbf{Q}}$ se plonge dans une $\mathbf{Q}$-variété projective lisse $Y_{\mathbf{Q}}$. On peut épaissir $Y_{\mathbf{Q}}$ en un schéma projectif lisse $Y$ sur $U$, où $U$ est un ouvert convenable de Spec $\mathbf{Z}$. L'application birationnelle de $X_{\mathbf{Q}}$ vers $Y_{\mathbf{Q}}$ définie par la discussion qui précède s'étend en une application birationnelle de $X$ vers $Y$. D'après le lemme 2, il suffit de prouver que $\operatorname{Pic}(Y)$ est de type fini.

Soient $h: Y \rightarrow U$ la projection, $V$ le normalisé de $U$ dans $g$ et $h^{\prime}: Y \rightarrow V$ le morphisme correspondant. Soit $V^{\prime}$ un ouvert de $V$ et $Y^{\prime}=h^{\prime-1}\left(V^{\prime}\right)$. Puisque $h^{\prime}$ est propre et à fibres géométriquement connexes, dans le diagramme commutatif aux lignes exactes

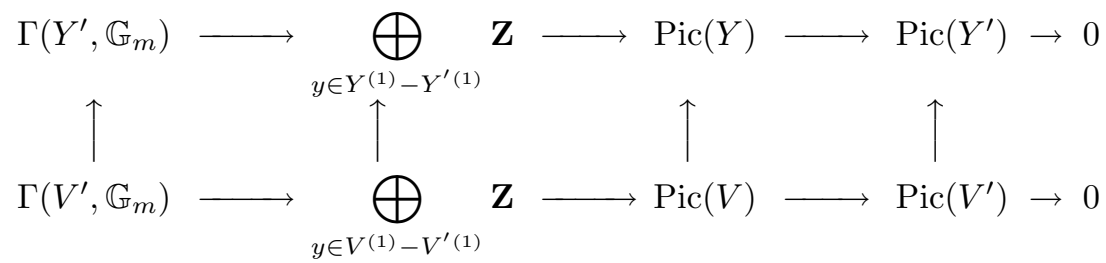

les deux flèches verticales de gauche sont des isomorphismes. On en déduit une suite exacte "de Mayer-Vietoris"

$$
0 \rightarrow \operatorname{Pic}(V) \longrightarrow \operatorname{Pic}(Y) \oplus \operatorname{Pic}\left(V^{\prime}\right) \longrightarrow \operatorname{Pic}\left(Y^{\prime}\right) \rightarrow 0 .
$$

En passant à la limite sur $V^{\prime}$, on en déduit une suite exacte :

$$
0 \rightarrow \operatorname{Pic}(V) \longrightarrow \operatorname{Pic}(Y) \longrightarrow \operatorname{Pic}\left(Y_{\mathbf{Q}}\right) \rightarrow 0 .
$$

Mais le groupe $\operatorname{Pic}\left(Y_{\mathbf{Q}}\right)$ est de type fini par une nouvelle application du lemme 4 et $\operatorname{Pic}(V)$ est de type fini (en fait, fini) comme groupe des classes d'un anneau de $S$-entiers de corps de nombres, ce qui conclut.

REMARque 3. - Au lieu d'utiliser la résolution des singularités dans le cas 2), on aurait pu se contenter du théorème de de Jong [17, th. 8.2], plus élémentaire, en utilisant la proposition 1 comme dans le cas 1 ). 
Corollaire 4. - La conclusion du lemme 4 s'étend au cas où $k$ est de type fini sur son sous-corps premier.

Il suffit d'insérer le corollaire 3 dans le raisonnement de la démonstration du lemme 4 .

\section{Démonstration du théorème 3}

Elle est de la même eau que la précédente. Nous démontrerons en même temps :

Proposition 2. - Sous les hypothèses du théorème 3, le noyau et le conoyau de la multiplication par $n$

$$
\mathrm{CH}_{d-1}(X) \stackrel{n}{\longrightarrow} \mathrm{CH}_{d-1}(X)
$$

sont finis pour tout $n \geq 1$.

LEMME 5. - Le théorème 3 et la proposition 2 sont vrais si $X$ est projectif et lisse.

On peut supposer que $X$ est purement de dimension $d$ : alors cela découle immédiatement du théorème 5 et de la représentabilité de $\operatorname{Pic}^{0}(X)$.

Lemme 6. - Soit $U$ un ouvert dense de X. Alors le théorème 3 et la proposition 2 sont vrais pour $X$ si et seulement si ils le sont pour $U$.

Cela résulte immédiatement de la suite exacte

$$
Z_{d-1}(Z) \longrightarrow \mathrm{CH}_{d-1}(X) \longrightarrow \mathrm{CH}_{d-1}(U) \rightarrow 0
$$

où $Z=X-U$ (de dimension $\leq d-1)$, puisque le groupe de gauche est de type fini.

Lemme 7. - Supposons $X$ lisse de dimension $d$. Soit $f: Y \rightarrow X$ un revêtement étale galoisien de groupe $G$. Si le théorème 3 et la proposition 2 sont vrais pour $Y$, ils le sont pour $X$.

Démonstration. - Traitons d'abord le cas de la proposition 2. Pour cela, considérons le complexe de $G$-modules

$$
C=\operatorname{cone}\left(\tau_{\leq 1} \mathbf{R} \Gamma\left(Y, \mathbb{G}_{m}\right) \stackrel{n}{\longrightarrow} \tau_{\leq 1} \mathbf{R} \Gamma\left(Y, \mathbb{G}_{m}\right)\right)
$$

où $\mathbf{R} \Gamma$ est pris pour la topologie étale. (Plus précisément, on choisit un complexe de $G$-modules représentant $\mathbf{R} \Gamma\left(Y, \mathbb{G}_{m}\right)$, par exemple les sections globales de la résolution de Godement de $\mathbb{G}_{m}$, et on lui applique la construction ci-dessus.) On a donc une suite exacte de $G$-modules

$$
\begin{aligned}
0 \rightarrow H^{-1}(C) \longrightarrow & \Gamma\left(Y, \mathbb{G}_{m}\right) \stackrel{n}{\longrightarrow} \Gamma\left(Y, \mathbb{G}_{m}\right) \\
& \rightarrow H^{0}(C) \longrightarrow \operatorname{Pic}(Y) \stackrel{n}{\longrightarrow} \operatorname{Pic}(Y) \longrightarrow H^{1}(C) \rightarrow 0 .
\end{aligned}
$$

TOME $134-2006-\mathrm{N}^{\mathrm{O}} 3$ 
On en déduit que les $H^{i}(C)$ sont finis : cela résulte de l'hypothèse et du lemme 1 qui implique que $\Gamma\left(Y, \mathbb{G}_{m}\right)$ est extension d'un groupe de type fini par $k^{*}$. Il en résulte que les groupes de cohomologie de $\mathbf{R} \Gamma(G, C)$ sont finis. Considérons maintenant le complexe (calcul dans la catégorie dérivée)

$$
\begin{aligned}
D=\tau_{\leq 1} \mathbf{R} \Gamma\left(G, \tau_{\leq 1} \mathbf{R} \Gamma\left(Y, \mathbb{G}_{m}\right)\right) & \\
\stackrel{\sim}{\longrightarrow} & \tau_{\leq 1} \mathbf{R} \Gamma\left(G, \mathbf{R} \Gamma\left(Y, \mathbb{G}_{m}\right)\right) \simeq \tau_{\leq 1} \mathbf{R} \Gamma\left(X, \mathbb{G}_{m}\right) .
\end{aligned}
$$

D'une part, on a

$$
H^{0}(D)=\Gamma\left(X, \mathbb{G}_{m}\right), \quad H^{1}(D)=\operatorname{Pic}(X), \quad H^{i}(D)=0 \quad \text { si } i \neq 0,1 .
$$

D'autre part, on a un triangle exact

$$
\mathbf{R} \Gamma\left(G, \tau_{\leq 1} \mathbf{R} \Gamma\left(Y, \mathbb{G}_{m}\right)\right) \stackrel{n}{\longrightarrow} \mathbf{R} \Gamma\left(G, \tau_{\leq 1} \mathbf{R} \Gamma\left(Y, \mathbb{G}_{m}\right)\right) \longrightarrow \mathbf{R} \Gamma(G, C) \stackrel{+1}{\longrightarrow}
$$

d'où une suite exacte

$$
\begin{aligned}
0 \rightarrow H^{-1}(\mathbf{R} \Gamma(G, C)) \longrightarrow H^{0}(D) \stackrel{n}{\longrightarrow} H^{0}(D) \\
\quad \rightarrow H^{0}(\mathbf{R} \Gamma(G, C)) \longrightarrow H^{1}(D) \stackrel{n}{\longrightarrow} H^{1}(D) \longrightarrow H^{1}(\mathbf{R} \Gamma(G, C))
\end{aligned}
$$

qui montre bien que le noyau et le conoyau $\operatorname{de} \operatorname{Pic}(X) \stackrel{n}{\longrightarrow} \operatorname{Pic}(X)$ sont finis.

Passons maintenant au cas du théorème 3. D'abord, l'argument de transfert habituel montre que le noyau de $A_{d-1}(X) \rightarrow A_{d-1}(Y)$ est annulé par $n=|G|$ : ainsi, l'hypothèse implique que $A_{d-1}(X)$ est extension d'un groupe de type fini par un groupe $E$ d'exposant $n$. En particulier, le noyau de $E \rightarrow A_{d-1}(X) / n$ est fini ; mais $A_{d-1}(X) / n$ est un quotient de $\operatorname{Pic}(X) / n$ qui est fini d'après la première partie de la démonstration. On en conclut que $E$ est fini et donc que $A_{d-1}(X)$ est de type fini.

Démonstration de la proposition 2 et du théorème 3. - On commence par se ramener au cas où $X$ est intègre. On utilise alors la version équivariante du théorème de de Jong [17, th. 8.1] : celle-ci implique qu'il existe un ouvert $U$ de $X$, qu'on peut supposer lisse, et un revêtement galoisien $f: Y \rightarrow U$ comme dans le lemme 7 , où $Y$ est un ouvert d'une variété projective lisse. Il suffit alors d'appliquer les lemmes 5, 6 et 7 .

\section{REMARQUeS 4}

1) Si $n$ est premier à la caractéristique de $k$ dans la proposition 2 , on peut remplacer le recours au théorème de de Jong par l'utilisation de l'homologie de Borel-Moore étale et des théorèmes de finitude de Deligne [6], Cycle §2.3 et version homologique de théorème de finitude, corollaire 1.10.

2) Le rapporteur a demandé s'il existe une version "uniforme" de la proposition 2, c'est-à-dire si l'ordre du noyau et du conoyau sont bornés par $n^{c}$, où $c$ est une constante ne dépendant que de $X$. Si $X$ est projective lisse, c'est clair étant donné la structure de $\operatorname{Pic}(X)$. On se convainc que c'est vrai en général 
en partant de ce cas et en suivant la stratégie de démonstration ci-dessus : le cas le plus désagréable est celui du lemme 7. (On remarquera qu'on obtient une constante $c$ différente selon que $n$ est premier à l'exposant caractéristique ou que c'est une puissance de celui-ci.)

\section{Démonstration du théorème 4}

On peut supposer $L \neq K$. Soient $\left(t_{1}, \ldots, t_{n}\right)$ une base de transcendance de $L$ sur $K, M_{0}=K\left(t_{1}, \ldots, t_{n-1}\right)$ et $M$ la fermeture algébrique de $M_{0}$ dans $L$. Alors $L / M$ et $M / K$ vérifient les hypothèses du théorème 4 ( $c f .[3, \S 14$, cor. 3, p. 113] ; par récurrence sur le degré de transcendance de $L / K$, on se ramène ainsi au cas où celui-ci est égal à 1 .

Soit $X$ la $K$-courbe projective régulière, géométriquement irréductible par hypothèse, de corps des fonctions $L$. Quitte à faire une extension finie radicielle de $K$ et à normaliser, on peut supposer $X$ lisse sur $K$. Comme $\operatorname{Br}(X)$ s'injecte dans $\operatorname{Br}(L)$, le noyau ci-dessus est le même que $\operatorname{Ker}(\operatorname{Br}(K) \rightarrow \operatorname{Br}(X))$. Or la suite spectrale de Hochschild-Serre donne une suite exacte

$$
\operatorname{Pic}(\bar{X})^{G} \longrightarrow \operatorname{Br}(K) \longrightarrow \operatorname{Br}(X) \text {. }
$$

Notons d'abord que si $X$ a un point rationnel, l'application $\operatorname{Br}(K) \rightarrow \operatorname{Br}(X)$ admet une rétraction, donc est injective; on en déduit par un argument de transfert qu'en général $\operatorname{Ker}(\operatorname{Br}(K) \rightarrow \operatorname{Br}(L))$ est d'exposant fini. Il suffit donc de montrer que pour tout $m>0$, premier à $p$ dans le cas (ii), le groupe $\operatorname{Pic}(\bar{X})^{G} / m$ est fini.

Dans le cas (i), la conclusion suit du corollaire 4. Dans le cas (ii), la suite exacte

$$
0 \rightarrow \operatorname{Pic}^{0}(\bar{X})^{G} \longrightarrow \operatorname{Pic}(\bar{X})^{G} \longrightarrow \mathbf{Z}
$$

nous ramène à voir que $\operatorname{Pic}^{0}(\bar{X})^{G} / m=P_{X}(K) / m$ est fini. Ceci n'est autre que le théorème de Mordell-Weil faible dont nous rappelons une démonstration : on peut trouver un schéma abélien $f: \mathcal{P} \rightarrow S$ dont la base $S$ est un modèle lisse de $K / k$ et dont la fibre générique est $P_{X}$, cf. [25, rem. 20.9]. On a alors, quitte à remplacer $S$ par un ouvert convenable ${ }^{(3)}$

$$
P_{X}(K)=\Gamma(S, \mathcal{P}) .
$$

La théorie de Kummer (c'est-à-dire la suite exacte $0 \rightarrow{ }_{m} \mathcal{P} \rightarrow \mathcal{P} \stackrel{m}{\rightarrow} \mathcal{P} \rightarrow 0$ de faisceaux étales) fournit une injection

$$
\Gamma(S, \mathcal{P}) / m \hookrightarrow H_{\text {ét }}^{1}\left(S,{ }_{m} \mathcal{P}\right) .
$$

D'autre part, on a une suite exacte (découlant de la suite spectrale de Hochschild-Serre ou plus simplement d'un calcul direct)

$$
H^{1}\left(k, H^{0}\left(\bar{S},{ }_{m} \mathcal{P}\right)\right) \rightarrow H_{\text {ét }}^{1}\left(S,{ }_{m} \mathcal{P}\right) \longrightarrow H^{0}\left(k, H^{1}\left(\bar{S},{ }_{m} \mathcal{P}\right)\right)
$$

${ }^{(3)}$ Ceci n'est en fait pas nécessaire.

TOME $134-2006-\mathrm{N}^{\mathrm{O}} 3$ 
où $\bar{S}=S \otimes_{K} \bar{k}$. Comme le faisceau ${ }_{m} \mathcal{P}$ est localement constant constructible, les modules galoisiens $H^{0}\left(\bar{S},{ }_{m} \mathcal{P}\right)$ et $H^{1}\left(\bar{S},{ }_{m} \mathcal{P}\right)=\operatorname{Hom}\left(\pi_{1}(\bar{S}),{ }_{m} \mathcal{P}\right)$ sont finis et on conclut. ${ }^{(4)}$

\section{Appendice A $K / k$-trace et $K / k$-image}

Dans cet appendice, on reprend la théorie de la $K / k$-trace et de la $K / k$-image de Chow d'un point de vue moderne et fonctoriel.

Pour tout corps $k$, notons $\mathrm{Ab}(k)$ la catgéorie des $k$-variétés abéliennes, les morphismes étant les homomorphismes de variétés abéliennes. D'autre part, notons $\operatorname{Gr}(k)$ la catégorie des $k$-groupes algébriques commutatifs (morphismes $=k$-homomorphismes) $: \mathrm{Ab}(k)$ est une sous-catégorie pleine de $\operatorname{Gr}(k)$.

LEMME 8. - Le foncteur d'inclusion $\mathrm{Ab}(k) \rightarrow \operatorname{Gr}(k)$ admet un adjoint à droite $G \mapsto G_{\mathrm{ab}}$, qui en est aussi un inverse à gauche.

Démonstration. - Soit $G \in \operatorname{Gr}(k)$. Comme tout quotient d'une variété abélienne par un sous-groupe fermé est une variété abélienne, $G$ admet une sousvariété abélienne maximale $G_{\mathrm{ab}}$, à savoir la somme de ses sous-variétés abéliennes. Tout $k$-homomorphisme $A \rightarrow G$, où $A$ est une variété abélienne, a son image dans $G_{\mathrm{ab}}$. Ceci montre que $G \mapsto G_{\mathrm{ab}}$ est adjoint à droite de l'inclusion. Si $G$ est une variété abélienne, on a évidemment $G_{\mathrm{ab}}=G$, ce qui montre la deuxième assertion.

REMARQUE 5. - La démonstration donne que la coünité de cette adjonction est toujours un monomorphisme.

Lemme 9. - Soient $K / k$ une extension finie radicielle et $A$ une $K$-variété abélienne. Alors il existe une $k$-variété abélienne $B$ et une isogénie radicielle $A \rightarrow B_{K}$.

Démonstration ( $c f .\left[18\right.$, p. 27]). — Pour tout $k$-schéma $X$, soit $F_{X}$ le Frobenius absolu de $X$. Soit $p^{n}=[K: k]$. Le Frobenius relatif

$$
A \longrightarrow\left(F_{K}^{n}\right)^{*} A
$$

induit par $F_{A}^{n}$ est une isogénie radicielle. Le lemme s'ensuit puisque $F_{K}^{n}$ se factorise à travers $k$.

Proposition 3 (Chow). — Soit $K / k$ une extension primaire, c'est-à-dire que le corps des constantes de $K$ est une extension radicielle de $k$. Soit $A$ une $k$ variété abélienne. Alors toute sous-variété abélienne $B$ de $A_{K}$ est définie sur $k$.

${ }^{(4)}$ Naturellement, on pourrait adapter cette démonstration également dans le cas (i) en faisant intervenir un localisé convenable de l'anneau des entiers de $k$. 
Démonstration. - On se réduit immédiatement au cas où $K / k$ est de type fini. Dans ce cas cela résulte de $[18$, p. 26 , th. 5$]$. Lorsque $K / k$ est régulière, on peut consulter [25, cor. 20.4, (a)] pour une démonstration en termes schématiques. Pour avoir une démonstration du cas général dans ce style, il reste à traiter celui où $K / k$ est finie radicielle. Dans ce cas nous proposons l'esquisse suivante, suggérée par le rapporteur :

Soit $R=K \otimes_{k} K$ : c'est une $K$-algèbre artinienne locale de corps résiduel $K$, donné par la multiplication $\mu: R \rightarrow K$. Les deux sous-schémas abéliens $K \otimes_{k} B$ et $B \otimes_{k} K$ de $A_{R}$ ont même restriction via $\mu^{*}$; un lemme de rigidité dont une démonstration utilise la densité de la $\ell$-torsion pour $\ell$ premier à $p$ implique alors qu'ils sont égaux. La conclusion en découle, par descente fidèlement plate.

(On pourrait bien sûr faire ce raisonnement directement quand $K / k$ est primaire et de type fini, en considérant un modèle $S$ de $K$ sur lequel $B$ s'étend en un schéma abélien, le point étant que $S \times_{k} S$ est connexe.)

Corollaire 5. - Si l'extension $K / k$ est primaire, le foncteur d'extension des scalaires $\mathrm{Ab}(k) \rightarrow \mathrm{Ab}(K)$ est pleinement fidèle.

Démonstration ( $c f$. [25, cor. 20.4, (b)]. — La fidélité est claire et la plénitude découle de la proposition 3 appliquée au graphe d'un morphisme.

Soit $K / k$ une extension de type fini et notons $f: \operatorname{Spec} K \rightarrow \operatorname{Spec} k$ la projection correspondante. Le théorème suivant est dû essentiellement à Chow.

THÉORÈme 6 . - Le foncteur d'extension des scalaires $\mathrm{Ab}(k) \rightarrow \mathrm{Ab}(K)$ admet un adjoint à gauche $f_{!}$et un adjoint à droite $f_{*}$. Lorsque $K / k$ est primaire, on appelle $f_{\text {! }}$ la $K / k$-image et $f_{*}$ la $K / k$-trace.

Nous allons en donner une démonstration qui a l'avantage d'expliciter les foncteurs $f_{\text {! }}$ et $f_{*}$.

Démonstration. - Comme les catégories $\mathrm{Ab}(k)$ et $\mathrm{Ab}(K)$ sont munies de dualités commutant à l'extension des scalaires, il suffit de démontrer l'existence de l'adjoint d'un côté (on a un isomorphisme fonctoriel

$$
\left(f_{!} A\right)^{\vee} \simeq f_{*}\left(A^{\vee}\right)
$$

où $\vee$ désigne le passage à la variété duale). De plus, si l'existence est démontrée pour une extension $K / k$ et pour une extension $M / K$, alors elle est démontrée pour l'extension $M / k$.

a) Supposons $K / k$ finie. On a alors le foncteur de restriction des scalaires (à la Weil) $R_{K / k}$, défini sur toutes les $K$-variétés quasi-projectives et adjoint à droite de l'extension des scalaires ( $c f$. par exemple $[33$, cor. 4.8.1, p. 38]). Notons qu'en tant qu'adjoint à droite, $R_{K / k}$ commute aux produits et est donc également adjoint à droite de l'extension des scalaires dans les catégories $\operatorname{Gr}(k)$ et $\operatorname{Gr}(K)$. Il est immédiat que $f_{*} A=R_{K / k}(A)_{\text {ab }}$ (composé de deux adjoints à droite).

TOME $134-2006-\mathrm{N}^{\mathrm{O}} 3$ 
b) Supposons maintenant $k$ algébriquement fermé dans $K$. Nous allons montrer l'existence de $f$ ! en la réduisant à l'existence de la variété d'Albanese. Pour cela, choisissons un modèle $X$ de $K / k$ muni d'un morphisme projectif lisse $p: \mathcal{A} \rightarrow X$, de fibre générique $A$. Soit $(B, \phi)$ (resp. $(C, \psi))$ "la" $k$-variété d'Albanese de $\mathcal{A}$ (resp. de $X$ ) au sens birationnel [18, p. 46], et soit $p_{*}: B \rightarrow C$ l'homomorphisme défini par $p$. Nous allons voir que

$$
f_{!} A=\operatorname{Ker}\left(p_{*}\right) .
$$

(Noter que $\operatorname{Ker}\left(p_{*}\right)$ est connexe puisque $p$ admet une section).

D'abord, soit $F$ le pull-back du diagramme



où $\delta_{X}$ est l'inclusion diagonale. La fibre générique de $\pi$ est $A \times_{K} A$. La composition $p_{*} \phi \rho$ est nulle; l'application rationnelle $\phi \rho$ induit donc une application rationnelle $F \rightarrow \operatorname{Ker}\left(p_{*}\right)$, donc une application rationnelle $\lambda: F \rightarrow \operatorname{Ker}\left(p_{*}\right) \times_{k} X$ et finalement une application rationnelle $A \times_{K} A \rightarrow \operatorname{Ker}\left(p_{*}\right)_{K}$, qui se prolonge en un $K$-morphisme $A \times_{K} A \rightarrow \operatorname{Ker}\left(p_{*}\right)_{K}$ d'après [25, th. 3.1]. Ce $K$-morphisme $\lambda$ envoie $(0,0)$ sur 0 , donc est un homomorphisme. On définit

$$
\eta: A \longrightarrow \operatorname{Ker}\left(p_{*}\right)_{K}
$$

comme la restriction de $\lambda$ à $A \times\{0\}$.

Ensuite, soit $\alpha: A \rightarrow D_{K}$ un homomorphisme de variétés abéliennes, où $D$ est une $k$-variété abélienne. Quitte à rétrécir $X, \alpha$ s'étend en un morphisme

$$
\mathcal{A} \longrightarrow D \times_{k} X
$$

toujours noté $\alpha$. On en déduit (grâce au caractère birationnel de $B$ et $C$ ) un homomorphisme

$$
B \longrightarrow D \oplus C
$$

commutant à la projection sur $C$, d'où un morphisme

$$
\beta: \operatorname{Ker}\left(p_{*}\right) \longrightarrow D \text {. }
$$

On vérifie immédiatement que $\beta_{K}=\eta \circ \alpha$.

Les propositions suivantes redonnent les propriétés classiques de la $K / k$ trace et de la $K / k$-image.

Proposition 4. - Soit $A \in \mathrm{Ab}(k)$. Alors l'unité $\eta_{*}^{A}: A \rightarrow f_{*}\left(A_{K}\right)$ est un monomorphisme et la coünité $\varepsilon_{!}^{A}: f_{!}\left(A_{K}\right) \rightarrow A$ est un épimorphisme de noyau connexe. Si $K / k$ est primaire, ce sont des isomorphismes.

BULletin DE LA SOCiÉtÉ MATHÉMATIQUE DE FRANCE 
Démonstration. - Par dualité, il suffit de traiter le cas de $f_{*}$ : il suffit alors d'observer comme dans $[33,(4.2 .5)]$ que (comme dans toute adjonction) la composition

$$
A_{K} \stackrel{\left(\eta_{*}^{A}\right)_{K}}{\longrightarrow} f_{*}\left(A_{K}\right)_{K} \stackrel{\varepsilon_{*}^{A_{K}}}{\longrightarrow} A_{K}
$$

est l'identité. Dans le cas primaire, l'assertion résulte formellement du corollaire 5 .

Proposition 5. - Supposons $K / k$ primaire, et soit $A \in \mathrm{Ab}(K)$. Alors :

a) L'unité $\eta_{!}^{A}: A \rightarrow\left(f_{!} A\right)_{K}$ est un épimorphisme et la coünité $\varepsilon_{*}^{A}$ : $\left(f_{*} A\right)_{K} \rightarrow A$ est de noyau fini (mais pas toujours radiciel, cf. [18, p. 213]).

b) Il existe une isogénie $f_{*} A \rightarrow f_{!} A$ fonctorielle en $A$.

c) Si K/k est radicielle, $\eta_{!}^{A}$ et $\varepsilon_{*}^{A}$ sont des isogénies.

Démonstration. - a) Par dualité il suffit de traiter le cas de $\eta_{!}^{A}$ : cela résulte de la proposition 3 . Le b) résulte de a) et du corollaire 5. Preuve de c) : par dualité, il suffit de traiter le cas de $\varepsilon_{*}^{A}$. D'après Greenberg [10, p. 263] ( $c f$. aussi Milne [23, p. 178]), le $K$-schéma en groupes $G=\left(R_{K / k} A\right)_{K}$ est extension d'une variété abélienne $A^{\prime}$ de même dimension que $A$ par un groupe unipotent $U$. On a donc la situation suivante :



La sous-variété abélienne $G_{\text {ab }}$ intersecte $U$ en un sous-groupe fini ; l'homomorphisme composé $G_{\mathrm{ab}} \rightarrow A^{\prime}$ est donc de noyau fini unipotent. Soit $N=$ $\operatorname{dim} U(\operatorname{donc} N=d([K: k]-1)$, où $d=\operatorname{dim} A)$ : comme $p^{N} U=0$, la multiplication par $p^{N}$ induit un homomorphisme $A^{\prime} \rightarrow G$, qui se factorise à travers $G_{\mathrm{ab}}$. Le composé $A^{\prime} \rightarrow G_{\mathrm{ab}} \rightarrow A^{\prime}$ est la multiplication par $p^{N}$; en particulier, $G_{\mathrm{ab}} \rightarrow A^{\prime}$ est un épimorphisme, donc une isogénie radicielle. D'autre part, comme $\operatorname{Hom}(U, A)=0$, l'homomorphisme $G \rightarrow A$ se factorise à travers $A^{\prime}$. Enfin, le lemme 9 implique que $\varepsilon_{*}^{A}$ est un épimorphisme; comme $\operatorname{dim} A=\operatorname{dim} A^{\prime}$, on en conclut que dans la suite

$$
\left(f_{*} A\right)_{K} \longrightarrow G_{\mathrm{ab}} \longrightarrow A^{\prime} \rightarrow A
$$

TOME $134-2006-\mathrm{N}^{\mathrm{O}} 3$ 
la première flèche est un isomorphisme et les deux suivantes sont des isogénies.

Remarques 6. - a) D'après [18, cor. 2], le noyau de $\varepsilon_{*}^{A}$ est radiciel si l'extension $K / k$ est régulière.

b) $\mathrm{Si} K$ est une $k$-algèbre étale, le foncteur d'extension des scalaires $\operatorname{Gr}(k) \rightarrow \operatorname{Gr}(K)$ qui admet $R_{K / k}$ comme adjoint à droite a aussi un adjoint à gauche, également donné par $R_{K / k}$ : cela se voit d'abord dans le cas déployé, puis dans le cas général par descente à partir du cas déployé. Dans le cas des variétés abéliennes, on a $f_{*} A=f_{!} A=R_{K / k} A$ pour toute $A \in \mathrm{Ab}(K)$ : en effet, il suffit de vérifier que $R_{K / k}$ transforme $A$ en une variété abélienne, ce qui se voit en passant à la clôture séparable de $k[33,(4.11 .3)$ p. 41]. (Noter que dans ce cas, $\operatorname{dim} f_{*} A=\operatorname{dim} f_{!} A=[K: k] \operatorname{dim} A$.)

c) Dans la démonstration de la proposition $5 \mathrm{c}$ ), l'argument montre que $p^{N} G \subset\left(f_{*} A\right)_{K}$, donc que $R_{K / k} A / f_{*} A$ est unipotent. Par contre, $R_{K / k} A$ ne contient aucun sous-groupe affine connexe de dimension $>0:$ si $M$ en était un, on aurait

\section{Contradiction!}

$$
\operatorname{Hom}_{k}\left(M, R_{K / k} A\right)=\operatorname{Hom}_{K}\left(M_{K}, A\right)=0 .
$$

Pour compléter notre exposé, donnons le résultat suivant qui indique que la formation de la $K / k$-trace et de la $K / k$-image commutent dans une certaine mesure à l'extension des scalaires.

Proposition 6. - Soient $K / k$ une extension de type fini, $A \in \mathrm{Ab}(K)$ et soit $L / k$ une extension linéairement disjointe de $K / k$. Notons $f^{\prime}: \operatorname{Spec} K L \rightarrow$ Spec L. Alors les morphismes de changement de base

$$
\left(f_{*} A\right)_{L} \longrightarrow f_{*}^{\prime}\left(A_{K L}\right), \quad f_{!}^{\prime}\left(A_{K L}\right) \longrightarrow\left(f_{!} A\right)_{L}
$$

sont des isogénies radicielles. Si L/k est séparable, ce sont des isomorphismes.

Esquisse de démonstration. - Par dualité il suffit de traiter le cas de $f_{!}$. Pour ce cas, cf. [18, p. 202, dém. du th. VIII.1.2] dont nous résumons le principe : on se réduit à $L / k$ de type fini, de plus si l'énoncé est vrai pour $L^{\prime} / k$ et $L / L^{\prime}$ alors il l'est pour $L / k$. Si l'extension $L / k$ est finie radicielle, l'énoncé résulte du lemme 9 ; si elle est séparable, il résulte de la théorie de la descente.

\section{Appendice B}

\section{Preuve du théorème 2 par Marc Hindry}

Nous donnons une preuve, rédigée dans le langage des schémas, du théorème 2 dî à Lang et Néron : si K/ $k$ est une extension de type fini régulière et $A$ est une $K$-variété abélienne, alors le groupe $A(K) / \tau B(k)$ est de type fini, où $\tau: B \rightarrow A$ est la $K / k$-trace de $A$. 
REMARQUe 7. - Il n'est pas difficile de démontrer un énoncé un peu plus général dans lequel on suppose seulement que $K / k$ est une extension de type fini obtenue par une extension algébrique séparable suivie d'une extension régulière et où l'on remplace la $K / k$-trace $B$ et l'homomorphisme à noyau fini $\tau: B_{K} \rightarrow A$ par la variété abélienne $f_{*} A$ et la coünité $\epsilon_{*}^{A}:\left(f_{*} A\right)_{K} \rightarrow A$ définies dans l'appendice précédent. En effet :

(i) L'énoncé pour $K / F$ et $F / k$ entraîne facilement celui pour $K / k$ (comparer avec le corollaire de la proposition 1 de [20]).

(ii) L'énoncé pour $K / k$ finie séparable est trivial puisque $\left(f_{*} A\right)(k)=$ $R_{K / k} A(k)=A(K)$.

Par contre j'ignore si l'énoncé pour $K / k$ finie purement inséparable reste vrai ou s'il existe des contre-exemples. Il ne semble pas suffisant d'utiliser le fait que $\epsilon_{*}^{A}:\left(f_{*} A\right)_{K} \rightarrow A$ est une isogénie d'après la proposition $\left.5, \mathrm{c}\right)$.

On suppose dorénavant que $K / k$ est régulière. Par récurrence sur le degré de transcendance de $K / k$, on se ramène au cas où ce degré est égal à 1 ; on peut également se ramener au cas où $k$ est algébriquement clos en observant que $A(K) / \tau B(k)$ s'injecte dans $A(\bar{k} K) / \tau B(\bar{k})$ car $A(K) \cap \tau(B(\bar{k}))=\tau(B(k))$, puisque $K / k$ est supposée régulière (comparer avec le corollaire de la proposition 2 de [20]). Alors $K$ est le corps des fonctions d'une courbe lisse projective $C$ définie sur $k$.

Notons $G:=A(K) / \tau B(k)$. On démontre l'analogue du théorème de Mordell-Weil faible dont la démonstration a été esquissée à la fin du paragraphe 3 : $G / m G \cong A(K) / m A(K)$ est fini, en choisissant $m \geq 2$ et premier à la caractéristique. Le point-clef est la proposition suivante qui permet par la méthode de la descente infinie de montrer que $G$ est engendré par un ensemble fini, où l'on a choisi une hauteur $h_{L}$ associée à un fibré (très) ample $L$ sur $A$.

Proposition 7. - L'ensemble $\left\{P \in A(K) \mid h_{L}(P) \leq c\right\}$ est fini modulo $\tau(B(k))$.

La hauteur $h_{L}$ peut être définie ainsi. On étend $A / K$ en un modèle $\pi$ : $\mathcal{A} \rightarrow C$ propre sur $C$ et sur lequel le fibré $L$ s'étend en un fibré $\mathcal{L}$ sur $\mathcal{A}$ (par exemple si $i: A \hookrightarrow \mathbf{P}_{K}^{n}$ avec $L=i^{*} O(1)$, on peut prendre pour $\mathcal{A}$ l'adhérence schématique de $A$ dans $\mathbf{P}_{C}^{n}$ et pour $\mathcal{L}$ la restriction à $\mathcal{A}$ de $\left.O(1)\right)$. Chaque point $P \in A(K)$ correspond à une section $P: C \rightarrow \mathcal{A}$ dont on note l'image $E_{P}$. On pose :

$$
h_{L}(P)=\operatorname{deg}_{C}\left(P^{*} \mathcal{L}\right) .
$$

Quitte à remplacer $\mathcal{L}$ par $\mathcal{L} \otimes \pi^{*}(\mathcal{M})$ (avec $\mathcal{M} \in \operatorname{Pic}(C)$ convenable) on peut supposer $\mathcal{L}$ (très) ample sur $\mathcal{A}$ et alors la théorie des coordonnées de Chow ( $c f$. [31], [26]) montre que l'ensemble des $E_{P}$ de hauteur bornée se trouve dans un nombre fini de familles algébriques (connexes ou irréductibles). En effet $\mathcal{L}$

TOME $134-2006-\mathrm{N}^{\mathrm{O}} 3$ 
correspond donc à un plongement $\mathcal{A} \hookrightarrow \mathbf{P}^{N}$ et dans ce plongement les $E_{P}$ telles que $h_{L}(P) \leq c$ sont des courbes de degré borné. Il reste donc à démontrer :

Lemme 10. - Deux sections $P_{1}, P_{2}$ appartenant à une même famille algébrique irréductible de sous-variétés de $\mathcal{A}$ sont congrues modulo $\tau(B(k))$.

Par hypothèse on dispose d'un "espace de paramètres" irréductible $T$, d'un cycle $Z \subset \mathcal{A} \times T \stackrel{\mathrm{pr}_{2}}{\longrightarrow} T$ et de deux points $t_{1}, t_{2} \in T(k)$ tels que $\mathrm{pr}_{2}^{-1}\left(t_{i}\right):=$ $Z_{t_{i}}=E_{P_{i}}$. En choisissant une courbe irréductible $T^{\prime}$ sur $T$ passant par $t_{1}, t_{2}$ (cf. [27, lemma p. 56]), en considérant sa normalisation $T^{\prime \prime} \stackrel{\nu}{\rightarrow} T^{\prime}$ et en remplaçant $Z$ par $Z^{\prime}:=(\text { id } \times \nu)^{-1}(Z)$ et $t_{i}$ par $t_{i}^{\prime} \in T^{\prime \prime}(k)$ tels que $\nu\left(t_{i}^{\prime}\right)=t_{i}$, on voit qu'on peut supposer que $T$ est une courbe lisse. On construit un morphisme $g$ de $T_{K}$ vers $A$ ainsi : l'intersection de $Z_{t}$ avec la fibre générique de $\mathcal{A}$ est un point qu'on note $g(t)$; en effet par construction $Z_{t_{i}}$ rencontre la fibre générique au seul point $P_{i}$, et cela reste donc vrai sur un ouvert dense. Ainsi $g$ est a priori une application rationnelle mais, comme $T$ est lisse et $A$ est une variété abélienne, il se prolonge en un morphisme. Soit $u: T \rightarrow \operatorname{Alb}(T)$ le morphisme d'Albanese (unique à une translation près par un élément de $\operatorname{Alb}(T)(k))$. Par la propriété universelle de la variété d'Albanese, l'application $g: T_{K} \rightarrow A$ induit un homomorphisme $\operatorname{Alb}(g): \operatorname{Alb}(T)_{K} \rightarrow A$ tel que $g$ coïncide avec $\operatorname{Alb}(g) \circ u$ à translation près par un élément de $A(K)$. Mais $\operatorname{Alb}(T)$ est une $k$-variété abélienne donc, par la propriété universelle de la $K / k$-trace, on en tire un homomorphisme $\beta: \operatorname{Alb}(T) \rightarrow B$ tel que $\operatorname{Alb}(g)=\tau \circ \beta$. On peut donc écrire :

$$
\begin{aligned}
P_{1}-P_{2}=g\left(t_{1}\right)-g\left(t_{2}\right)=\operatorname{Alb}(g) & \circ u\left(t_{1}\right)-\operatorname{Alb}(g) \circ u\left(t_{2}\right) \\
& =\tau \circ \beta \circ u\left(t_{1}\right)-\tau \circ \beta \circ u\left(t_{2}\right) \in \tau(B(k)) .
\end{aligned}
$$

Ce qui achève la preuve.

\section{BIBLIOGRAPHIE}

[1] Berthelot (P.), Grothendieck (A.) \& Illusie et Al. (L.) - Séminaire de géoàmétrie algébrique du Bois-Marie (SGA 6) : Théorie des intersections et théorème de Riemann-Roch, in Lecture Notes in Math., vol. 225, Springer, 1971.

[2] Bosch (S.), Lütkebohmert (W.) \& Raynaud (M.) - Néron models, Springer, 1990.

[3] Bourbaki (N.) - Éléments de mathématique, Algèbre, Ch. V, Masson, 1981.

[4] Chow (W. L.) - Abstract theory of the Picard and Albanese varieties, manuscrit non publié $(1955 / 56)$.

BULletin DE LA SOCiÉtÉ MATHÉMATIQUE DE FRANCE 
[5] Colliot-Thélène (J.-L.) \& SAnsuc (J.-J.) - La R-équivalence sur les tores, Ann. Sci. Éc. Norm. Sup., t. 10 (1977), pp. 175-229.

[6] Deligne et AL. (P.) - Séminaire de géométrie algébrique du Bois-Marie (SGA 41/2) : Cohomologie étale, in Lecture Notes in Math., vol.569, Springer, 1977.

[7] Demazure (M.) \& Grothendieck et AL. (A.) - Séminaire de géométrie algébrique du Bois-Marie (SGA 3) : Schémas en groupes, in Lecture Notes in Math., vol. II, Springer, 1970.

[8] Fein (B.), Kantor (W. M.) \& Schacher (M.) - Relative Brauer groups, II, J. reine angew. Math., t. 328 (1981), pp. 31-57.

[9] Fulton (W.) - Intersection theory, Springer, 1984.

[10] Greenberg (M. J.) - Schemata over local rings, Ann. of Math., t. 78 (1963), pp. 256-266.

[11] Grothendieck (A.) - Techniques de descente et théorèmes d'existence en géométrie algébrique, in Fondements de la géométrie algébrique, extraits du Séminaire Bourbaki 1957-62, Secrétariat mathématique, Paris.

[12] _ Sur une note de Mattuck-Tate, J. reine angew. Math., t. 200 (1958), pp. 208-215.

[13] Grothendieck (A.) \& Dieudonné (J.) - Éléments de géométrie algébrique, chapitre IV : Étude locale des schémas et des morphismes de schémas (2e partie), Publ. Math. IHÉS, t. 24 (1965).

[14] Guralnick (R.), Jaffe (D.), Raskind (W.) \& Wiegand (R.) - On the Picard group : torsion and the kernel induced by a faithfully flat map, J. Algebra, t. 183 (1996), pp. 420-455.

[15] Hartshorne (R.) - Algebraic geometry, Springer, 1977.

[16] Hironaka (H.) - Resolution of singularities of an algebraic variety over a field of characteristic zero, Ann. of Math., t. 79 (1964), pp. 109-326.

[17] DE Jong (P. A.) - Smoothness, semi-stability and alterations, Publ. Math. IHÉS, t. 83 (1996), pp. 51-93.

[18] LANG (S.) - Abelian varieties, Interscience, 1957, reprint, Springer, 1983.

[19] _ Fundamentals of diophantine geometry, Springer, 1983.

[20] LANG (S.) \& NÉRON (A.) - Rational points of abelian varieties over function fields, Amer. J. Math., t. 81 (1959), pp. 95-118.

[21] Manin (Yu.) - The Mordell-Weil theorem, appendice II ̀̀ [27].

[22] Matsusaka (T.) - On the algebraic construction of the Picard variety, I, Japan J. Math., t. 21 (1951), pp. 217-235, II, ibid., 22 (1952), 51-62.

[23] Milne (J. S.) - On the arithmetic of abelian varieties, Invent. Math., t. 17 (1972), pp. 177-190.

[24] _ Étale cohomology, Princeton Univ. Press, 1980.

[25] - Abelian varieties, p. 103-150, in Arithmetic geometry (G. Cornell, J. Silverman, eds), Springer, 1986.

TOME $134-2006-\mathrm{N}^{\mathrm{O}} 3$ 
[26] Mumford (D.) - Geometric Invariant Theory, Springer-Verlag, 1965.

[27] _ Abelian varieties, Oxford University Press, 1970.

[28] Nagata (M.) - A generalization of the imbedding problem of an abstract variety in a complete variety, J. Math. Kyoto Univ., t. 3 (1963), pp. 89 102.

[29] NÉRON (A.) - Problèmes arithmétiques et géométriques rattachés à la notion de rang d'une courbe algébrique dans un corps, Bull. Soc. Math. France, t. 80 (1952), pp. 101-166.

[30] Roquette (P.) - Einheiten und divisorklassen in endlich erzeugbaren körpern, Jahresber. Deutsch. Math. Verein., t. 60 (1957), pp. 1-21.

[31] Samuel (P.) - Méthodes d'algèbre abstraite en géométrie algébrique, Springer-Verlag, 1955.

[32] _ À propos du théorème des unités, Bull. Sci. Math., t. 90 (1966), pp. 89-96.

[33] SCHEIDERER (C.) - Real and étale cohomology, in Lecture Notes in Math., vol. 1588, Springer, 1994.

[34] SEgRE (B.) - Intorno ad un teorema di hodge sulla teoria della base per le curve di una superficie algebrica, Ann. Mat., t. 16 (1937), pp. 157-163.

[35] SEVERI (F.) - Sui fondamenti della geometria numerativa e sulla teoria delle caratteristiche, Ist. veneto sci. lett. arti. Parte II, , t. 75, pp. 11211162, Opere mat. II, Acad. Nat. Lincei, Rome, LXVIII (1974), 500-530.

[36] _ Sulla totalitá delle curve algebriche tracciate sopra una superficie algebrica, Math. Ann., t. 62 (1906), pp. 194-225, Opere Mat. I, Acad. Nat. Lincei, Rome, XXIX (1971), 332-362. 